\section{SOLMIZAÇÃO: UM ESTUDO SISTEMÁTICO DE SEU ENSINO A PARTIR DE FONTES PRIMÁRIAS INGLESAS DO FINAL DO SÉCULO XVI ATÉ O FIM DO XVII.}

RESUMO: Este artigo aborda aspectos do ensino da Solmização à partir do estudo sistemático de fontes primárias inglesas do final do século XVI até o fim do XVII. Pretende-se evidenciar as particularidades do ensino da Solmização dentre os autores ingleses já que eles utilizam apenas quatro Sílabas: mi, fá, sol, lá. Para isto, será explicitado a forma como Thomas Morley (1597), Thomas Campion (ca. 1614), Charles Butler (1636), John Playford (1655) e Christopher Simpson (1667) tratam este assunto. Pode-se concluir que apesar da maioria dos tratados ingleses de música prática do século XVI até o fim do XVII serem baseados no sistema hexacordal, não explicam todas as regras necessárias para a sua compreensão.

PALAVRAS-CHAVE: teoria musical inglesa, Solmização, século XVI, século XVII

SOLMIZATION: a systematic study of its teaching from English primary sources from the late of sixteenth until the end of seventeenth centuries

ABSTRACT: This article covers the teaching of Solmization through a systematic study of English primary sources of the late sixteenth to the end of the seventeenth centuries. It aims to highlight the particularities of teaching Solmization among English authors since they use only four syllables: $m i$, fa, sol, la. For this purpose, it will be explained how Thomas Morley (1597), Thomas Campion (ca. 1614), John Playford (1655) and Christopher Simpson (1667) deal with this issue. It can be concluded that although most of English treatises of practical music from sixteenth century to the end of the seventeenth are based on the hexacordal system, they do not explain all the necessary rules for their understanding.

\author{
* Doutorando USP, E-mail: \\ daimon@usp.br. \\ Departamento de Música \\ - ECA/USP - \\ nathaliadomingos@yahoo. \\ com.br \\ Auxílio FAPESP (Mestrado)
}


KEYWORDS: English music theory, Solmization, sixteenth-century, seventeenth-century

\section{INTRODUÇÃO}

O presente artigo aborda aspectos do ensino da Solmização a partir do estudo sistemático de fontes primárias inglesas do final do século XVI até o fim do XVII. O ensino musical daquela época pode ser demonstrado através de manuais e tratados musicais práticos que permitem que o estudante aprenda a ler uma partitura, além de, muitas vezes, versarem sobre as regras da composição e do contraponto.

A maioria dos livros teóricos ingleses até o fim do século XVII são baseados no sistema hexacordal de Guido d'Arezzo, no entanto não expõem as regras necessárias para a sua compreensão. $\mathrm{O}$ único autor inglês que explica de forma completa o antigo sistema de Solmização foi Thomas Morley em seu $A$ Plaine and Easie Introduction to Practicall Musicke (1597).

Pretende-se com este artigo evidenciar as singularidades do ensino da Solmização pelos ingleses, já que utilizavam apenas quatro Sílabas: $m i$, fá, sol, lá. Para isto, será explicitado a forma como Thomas Morley (1597), Thomas Campion (ca. 1614), John Playford (1655) e Christopher Simpson (1667) tratam este assunto. A escolha destes autores se deve pelo fato de serem representativos e por abrangerem a delimitação cronológica proposta. A obra de Playford, por exemplo, teve sua décima e segunda edição publicada em 1694.

\section{SolmizaçÃo E MutaÇÃo - BreVe panorama}

De acordo com Mengozzi (2010, p. 1), por volta de 1032 o monge Guido d'Arezzo propôs um novo método para canto baseado em seis sílabas $u t$, ré, mi, fá, sol, lá que correspondiam às primeiras sílabas de cada verso do Hino a São João Ut queant laxis. Este método atribuído a Guido circulou por toda Europa e foi descrito em tratados musicais até o século XIX. No entanto a Solmização inglesa difere daquela praticada no continente europeu ${ }^{1}$, pois eles utilizavam apenas quatro sílabas de Solmização - mi, fá, sol, lá. 
A nomenclatura utilizada neste artigo baseia-se no tratado de Thomas Morley (1597). A seguir uma breve definição dos principais termos empregados:

- Dedução: sequência das seis Vozes musicais: ut, ré, mi, fá, sol, lá. Geralmente conhecido como hexacorde.

- Mutação: passar de uma Dedução para outra de acordo com sua necessidade. Mutação significa abandonar uma Voz e pegar outra do mesmo Signo, ou seja, com o mesmo som para solmizar acima do lá ou abaixo do ut.

- Propriedade: ligada à característica da nota "Si". Havia duas formas da letra "b" para diferenciar as duas alturas disponíveis para o "Si": b quadratum e b rotundum.

- Signo: uma palavra que contém em si o nome da letra e o(s) nome(s) da(s) Vozes. A combinação da letra ( $\Gamma, \mathrm{A}, \mathrm{B}$, C, D, F etc.) com a Voz (ut, ré, mi, fá, sol, lá) forma o nome composto do Signo, por exemplo: D sol ré.

- Voz: ferramenta que facilita a leitura de uma linha melódica à primeira vista, mas não indica a altura real daquela nota. Também conhecida como sílabas de Solmização: ut, ré, mi, fá, sol, lá.

A altura das notas era dada pelas letras $\Gamma$ A B C D E F (Fig. 1). Em geral, as canções ultrapassavam o âmbito das seis sílabas e o cantor/instrumentista deveria então fazer a Mutação. Para entender melhor este procedimento, devemos lembrar que as notas tinham mais de uma sílaba de Solmização associadas à elas.

Para exemplificar, vejamos na Fig. 1 que a nota Dó3, por exemplo, era denominada c sol fá ut. Cada uma dessas sílabas (sol fá $u t)$ pertencia a diferentes Deduções.

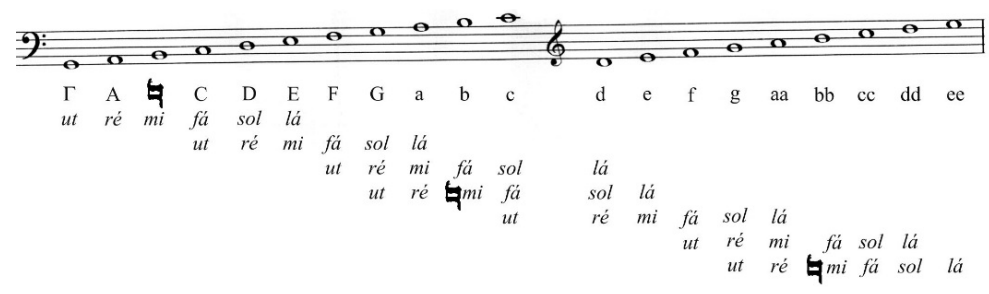

Figura 1: Altura exata das notas de acordo com suas letras e Vozes.

As Deduções seguem um padrão intervalar: T-T-St-T-T e são classificadas de acordo com três Propriedades: $b$ durum, naturalis e $b$ molle.
1. "Continente" é um termo genérico adotado neste artigo para se referir unicamente àqueles tratados citados por Thomas Morley durante a primeira parte do A plaine and Easie Introduction to Practicall Musicke, a saber: Aaron, Gaffurius, Glareanus, Listenius, Lossius, Ornithoparcus, Zacconi e Zarlino. 


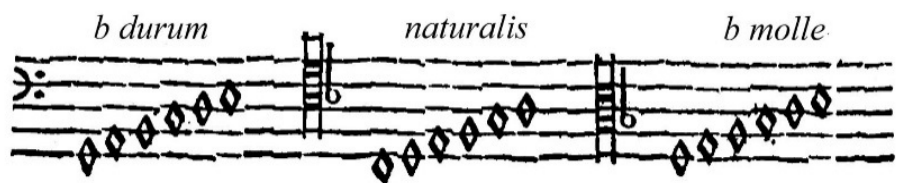

Figura 2: As três Propriedades (MORLEY, 1597, p. 4).

O meio tom pode ser encontrado entre as notas $\mathrm{Mi}$ - Fá, Lá - Si i e entre Si - Dó. Propriedade de $b$ durum porque tem sua origem no Signo $\Gamma$ ut e é quando o semitom está entre as notas $\mathrm{Si}$ - Dó. Naturalis é quando a Dedução tem o seu início no Signo C fá ut e o semitom se localiza entre as notas Mi - Fá, enquanto a Propriedade de $b$ molle tem sua origem no Signo F fá ut e o semitom encontra-se entre as notas Lá - Si

A Mutação ensinada nos tratados que circulavam no continente europeu consistia na seguinte fórmula: sempre ocorria na Voz ré para melodias ascendentes e na Voz lá para as melodias descendentes.

A seguir uma demonstração das Mutações na melodia em cantus durus (em vermelho na Tab. 1). No caso de não haver sinal de bemol na Clave, as Deduções em $b$ durum e naturalis são solicitadas.

Observe que a Mutação sempre ocorre na Voz ré da nova Dedução na melodia ascendente, enquanto na descendente ocorre na Voz lá da nova Dedução.

\begin{tabular}{|c|c|c|c|c|c|c|c|c|c|c|c|c|c|c|}
\hline ee & & & & & & & lá & lá & & & & & & \\
\hline $\mathrm{dd}$ & & & & & & $l a ́ a$ & sol & sol & 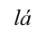 & & & & & \\
\hline $\mathrm{cc}$ & & & & & & sol & $f \ddot{a}$ & $f \dot{a}$ & sol & & & & & \\
\hline$b 6$ & & & & & & 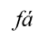 & $\forall m i$ & $4 m i$ & 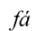 & & & & & \\
\hline aa & & & & & lá & $m i$ & $r e^{\prime}$ & $r e ́$ & $m i$ & lá & & & & \\
\hline $\mathrm{g}$ & & & & & sol & $r e ́$ & $u t$ & $u t$ & $r e ́$ & sol & & & & \\
\hline $\mathrm{f}$ & & & & & $f a ́$ & $u t$ & & & $u t$ & $f a ́$ & & & & \\
\hline $\mathrm{e}$ & & & & lá & $m i$ & & & & & $m i$ & $\boldsymbol{l a ́}$ & & & \\
\hline $\mathrm{d}$ & & & lá & sol & $r \dot{e}$ & & & & & $r e ́$ & sol & lá & & \\
\hline $\mathrm{c}$ & & & sol & $f \dot{a}$ & $u t$ & & & & & $u t$ & $f \dot{a}$ & sol & & \\
\hline$b$ & & & $f a ́$ & ษ $m i$ & & & & & & & $4 m i$ & $f a ́$ & & \\
\hline a & & lá & $m i$ & $r e ́$ & & & & & & & $r e ́$ & $m i$ & lá & \\
\hline G & & sol & $r e ́$ & $u t$ & & & & & & & $u t$ & $r e ́$ & sol & \\
\hline F & & $f a \dot{a}$ & $u t$ & & & & & & & & & $u t$ & $f \dot{a}$ & \\
\hline E & lá & $m i$ & & & & & & & & & & & $m i$ & lá \\
\hline D & sol & $r \dot{e}$ & & & & & & & & & & & $r e ́$ & sol \\
\hline C & $f \ddot{a}$ & $u t$ & & & & & & & & & & & $u t$ & 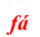 \\
\hline$\Rightarrow$ & $m i$ & & & & & & & & & & & & & $m i$ \\
\hline A & $r \dot{~}$ & & & & & & & & & & & & & $r e ́$ \\
\hline$\Gamma$ & $u t$ & & & & & & & & & & & & & $u t$ \\
\hline
\end{tabular}

Tabela 1: Mutação entre Deduções $b$ durum e naturalis praticada no continente - escala ascendente e descendente.

Caso haja o sinal da Clave 䤃 indicado no início do pentagrama, considera-se que a melodia esteja em cantus mollis e 
deve operar com as Deduções em $b$ molle e naturalis. O procedimento é idêntico: busca-se sempre a Voz ré da próxima Dedução para subir e para descer, a Voz lá (Tab. 2).

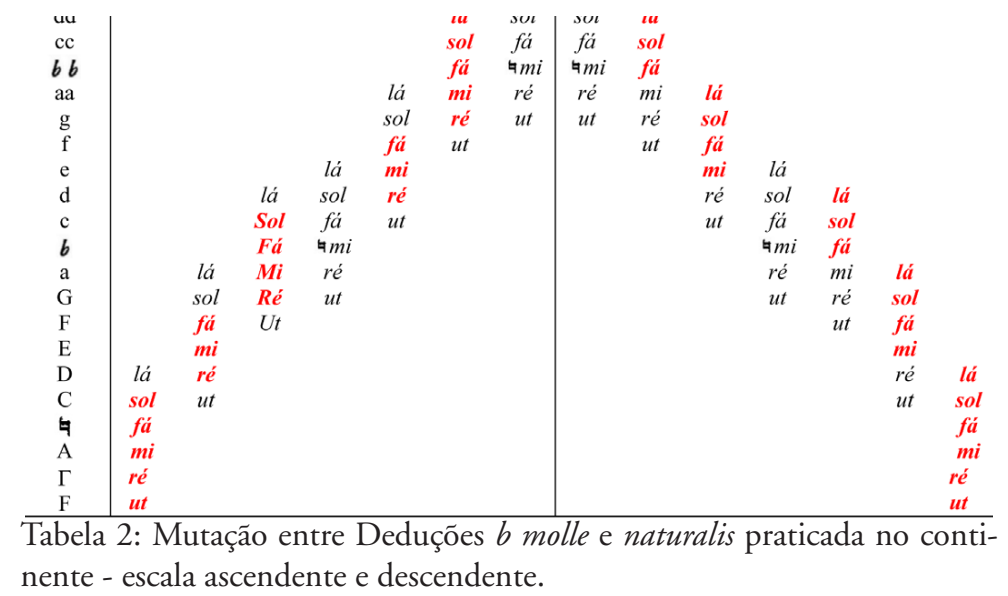

De acordo com Barnett (2002, p 436), a Solmização inglesa difere notadamente daquela praticada e ensinada no continente europeu, apesar de terem o mesmo fundamento. É possível encontrar entre os vários autores ingleses maneiras distintas de seu ensino.

A seguir, serão brevemente expostas as maneiras como Thomas Morley (1597), Thomas Campion (ca. 1614), John Playford (1655) e Christopher Simpson (1667) ensinam a Solmização.

\section{Thomas Morley (1597)}

Segundo Morley (1597, p. 4-5), a Dedução cuja Propriedade é de $b$ durum tem sua origem no Signo $\Gamma u t$. A Voz mi é sempre cantada no Signo $f a ́$ mi e seu reconhecimento se dá pela ausência da Clave no início do pentagrama.

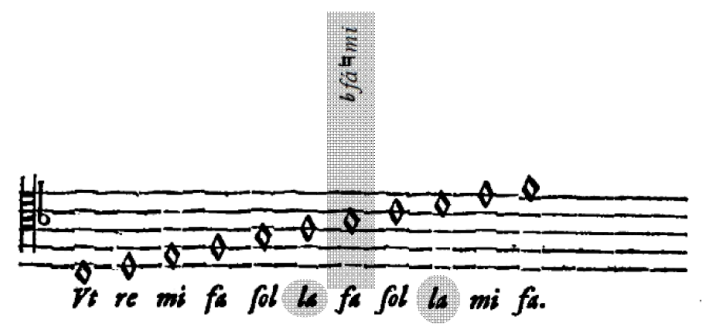

Figura 3: Propriedade de $b$ durum (MORLEY, 1597, p. 5). 
Já a Propriedade naturalis origina-se no Signo C fá ut. Pode-se cantar tanto a Voz fá quanto mi no Signo fá $m i$. Morley (1597, p. 203) esclarece que as três Propriedades são encontradas no cantochão, mas no canto figurado apenas duas são de fato usadas: $b$ molle e $\quad b$ durum. Segundo Owens (1998, p. 202), esta passagem sugere que Morley emprega a dicotomia durus-mollis da teoria continental fazendo com que as Mutações operem no cantus durus entre a Propriedade de $b$ dur:n n e naturalis, enquanto no cantus mollis, entre a Proprie$\mathrm{dad} \underset{\tilde{\text { I }}}{\tilde{\pi}} \mathrm{de} b$ molle e naturalis.

- O exemplo da Fig. 4 demonstra a Voz fá no Signo $f a ́$ 日 $m$.

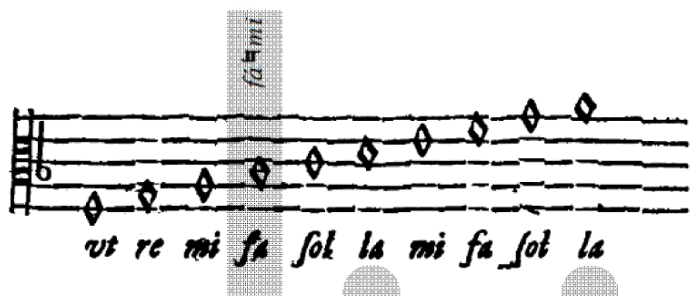

Figura 4: Propriedade naturalis em cantus mollis. (MORLEY, 1597, p. 6).

Já a Propriedade de $b$ molle é reconhecida pela Clave posicionada no início do pentagrama e tem sua origem no Signo F fá ut. A Voz fá deve ser pronunciada no Signo fá mi

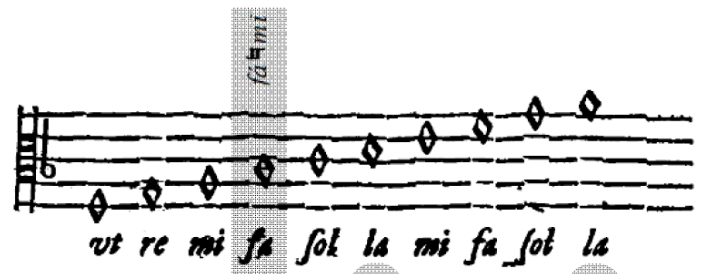

Figura 5: Propriedade de $b$ molle (MORLEY, 1597, p. 6).

Para Morley, a Mutação nas melodias ascendentes (cantus durus e cantus mollis) deve ocorrer quando se chega à Voz lá da Dedução atual, como pode ser observado em destaque nas Figuras 3, 4 e 5. Nas melodias descendentes, busca-se sempre o lá da nova Dedução para se fazer a Mutação. 


\section{Thomas Campion (Ca. 1614)}

Para Campion (ca. 1614, $f$ B4a), o verdadeiro conhecimento da escala consiste da observação do meio tom que é expressado pelas sílabas $m i$ - fá ou lá - fá. Além disso, Campion (ca. $1614, f \mathrm{~B} 4 \mathrm{v})$ chama atenção para que o indivíduo, além de observar as localizações dos semitons que são marcados pelos semicírculos, lembre-se que se o semitom inferior for $m i-f a ́$, o semitom superior será lá - fá e vice-versa.

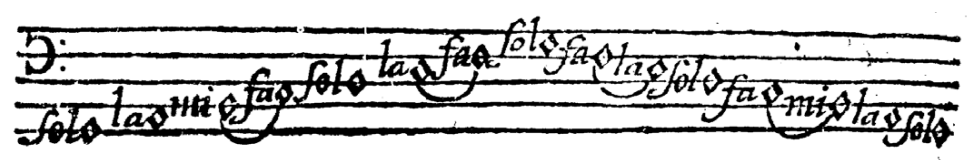

Figura 6: Escala durum (sharp) (CAMPION, ca. 1614, $f$ B4v).

O autor ainda dá uma regra geral para a nomeação das demais notas: acima de fá sempre cantar sol e abaixo de lá sempre cantar sol.

$\mathrm{Na}$ escala $b$ molle deve-se encontrar lá - fá na parte inferior e mi-fá na parte superior como pode ser observado na Fig. 7.

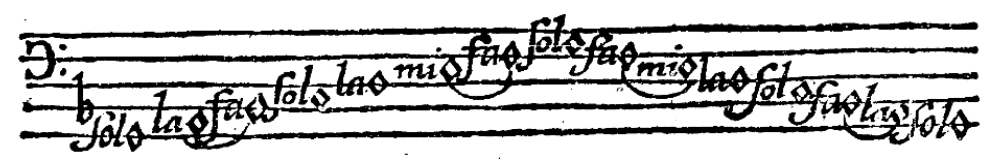

Figura 7: Escala $b$ molle (flat Gam-vt) (CAMPION, ca. 1614, $f$ B5a).

$\mathrm{Na}$ Fig. 8, encontramos um bemol no Signo E lá mi.

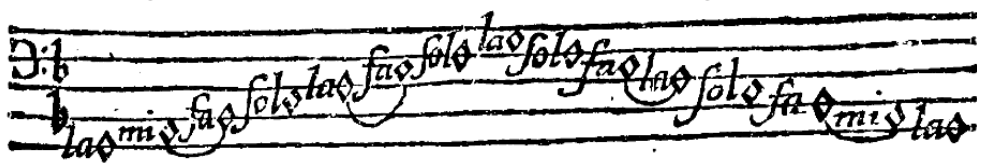

Figura 8: Escala com $b$ molle em E lá mi (CAMPION, ca. 1614, $f$ B5a).

\section{JoHn Playford (1655)}

Em seu An Introduction to the skill of Musick, Playford (1655, p. 11) estabelece regras para a localização da Voz mi.

A observação do $m i$ propicia o conhecimento das demais Vozes, visto que, segundo o autor, acima dele temos duas vezes a sequência fá-sol-lá até encontrar novamente a Voz mi, enquanto abaixo dele, duas vezes a sequência lá-sol-fá até se deparar de novo com o mi. 


$$
\begin{array}{r}
\text { MI - fá sol lá fá sol lá } \quad \text { MI fá sol láfá } \\
\text { sol lá- MI }
\end{array}
$$

Para Playford (1655, p. 11), o mi pode ser acomodado em quatro locais:

1. O local natural da Voz mi é no Signo $f^{-} f^{\prime} m i$.

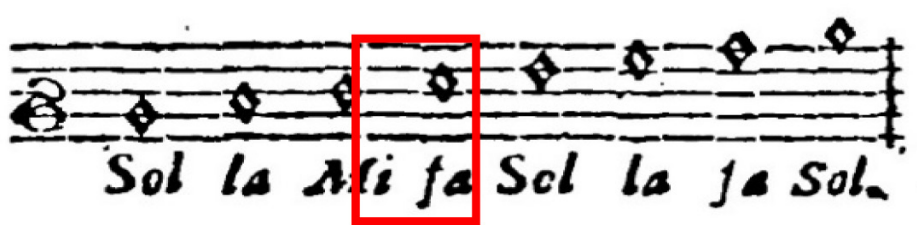

Figura 9: Posição natural do mi (PLAYFORD, 1655, p. 12).

2. Se o Signo $f a ́$ mi tiver um bemol, o mi deve ser acomodado no Signo E lá mi.

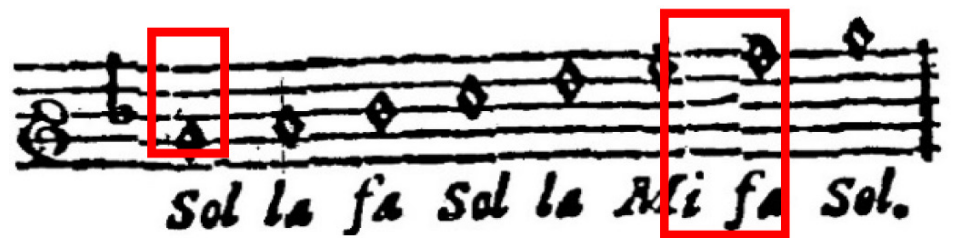

Figura 10: Segunda posição do mi (PLAYFORD, 1655, p. 13).

3. Se os Signos $f a ́$ mi e E lá mi tiverem o sinal de bemol, a Voz mi deve ser colocada no Signo a la mi ré.

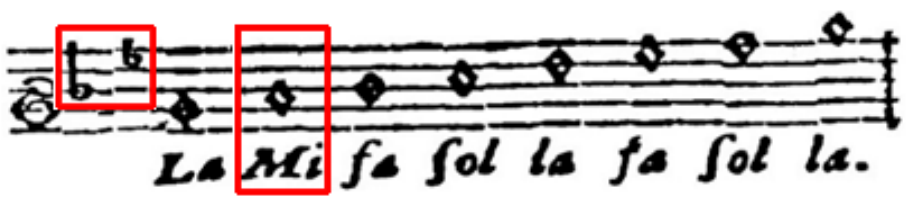

Figura 11: Terceira posição do mi (PLAYFORD, 1655, p. 13).

4. Se os Signos $f a ́$ mí E lá mi \& a lá mi ré tiverem o sinal de bemol na Clave, o $m i$ deve ser recolocado no Signo $\mathrm{D}$ lá sol ré.

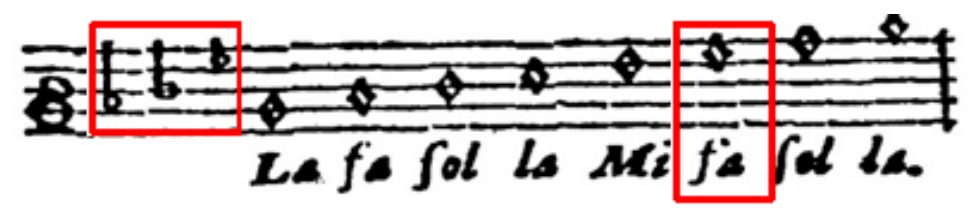

Figura 12: Quarta posição do mi (PLAYFORD, 1655, p. 13). 


\section{Christopher Simpson (1667)}

Para Simpson (1667, p. 5), devemos encontrar o mi, pois, conhecendo sua localização, as outras três sílabas são deduzidas sequencialmente já que acima dele temos fá-sol-lá e abaixo, lá-sol-fá.I (citação?)

$$
\mid \begin{aligned}
& l a \\
& f o l \\
& f a \\
& \hline M a \\
& \hline l a \\
& f o l \\
& f a
\end{aligned}
$$

Figura 13: Nomeação das demais Vozes a partir do mi (SIMPSON, 1667, p. 5).

Simpson também determina regras para a colocação da Voz mi:

1. O local natural da Voz mi é no Signo $f a ́$ mi.

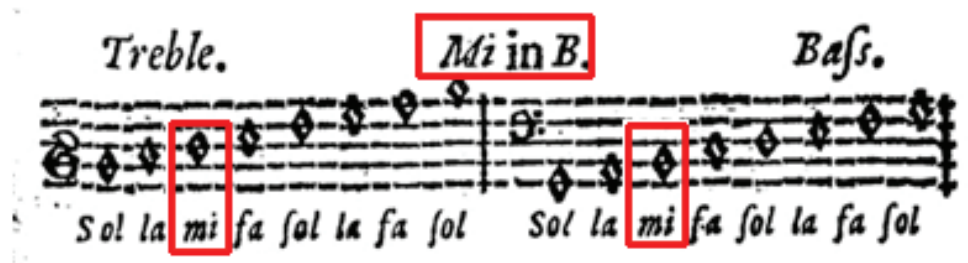

Figura 14: Posição natural do mi (SIMPSON, 1667, p. 11).

5. Se o Signo fá $m i$ tiver um bemol na Clave, então a Voz mi deve ser colocada no Signo E lá mi.

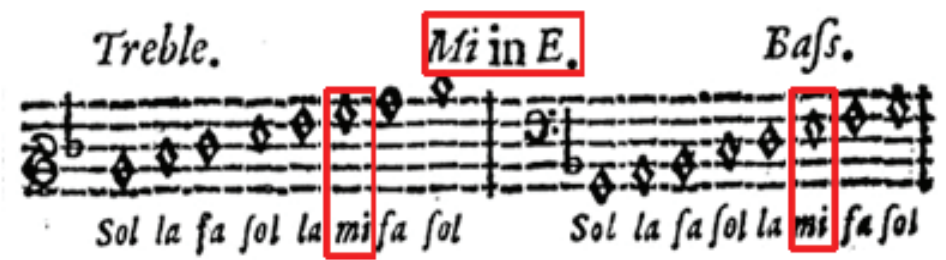

Figura 15: Segunda posição do mi (SIMPSON, 1667, p. 11).

6. Caso os Signos $f a ́$ mi e lá mi tenham um bemol, a Voz mi deve ser remanejada para o Signo a lá mi ré. 


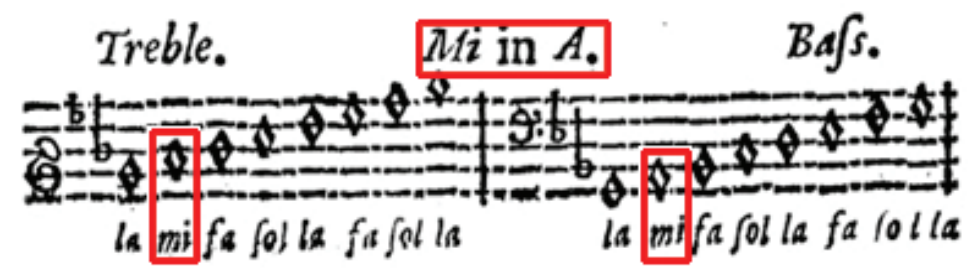

Figura 16: Terceira posição do mi (SIMPSON, 1667, p. 11).

\section{Consideraçốes Finais}

É possível encontrar entre os vários autores ingleses maneiras distintas do ensino da Solmização.

Thomas Morley (1597) é o único autor inglês que explica o antigo sistema de Solmização demonstrando as três Propriedades do canto, além de trazer exercícios para a prática do aprendiz. Para Morley, a Mutação nas melodias ascendentes se dá quando se chega à Voz lá da Dedução atual, enquanto nas melodias descendentes, busca-se sempre o lá da nova Dedução.

Campion (ca. 1614), Playford (1655) e Simpson (1667) não explicam o antigo sistema de Solmização e Mutação. Para eles o essencial é posicionar corretamente a Voz mi, pois desta forma as demais sílabas são deduzidas sequencialmente. Em geral, o mi podia ser acomodado em três locais: nos Signos 䝑 mi; E lá mi \& a lá mi ré.

Conclui-se que apesar da maioria dos tratados ingleses de música prática do século XVI até o fim do XVII serem baseados no sistema hexacordal, não explicavam todas as regras necessárias para a sua compreensão.

REFERÊNCIAS BiBLIOGRÁFICAS

BARNETT, G. Solmization and key in English theory. In: CHRISTENSEN, T. (Ed.). The Cambridge History of Western Music Theory. Cambridge: Cambridge University Press, 2002. cap. 13 , p. $435-441$

MENGOZZI, S. The Renaissance Reform of Medieval Music Theory: Guido of Arezzo between Myth and History. New York: Cambridge, 2010. 286p. 
MORLEY, Thomas. A Plaine and Easie Introduction to Practicall Musicke. London: Peter Short, 1597. Disponível em:

$<$ http:// e ebo.chadwyck.com/search/full rec?SOURCE=pgthumbs.cfg\&ACTION=ByID\&ID=99 847107\&FILE=../session/1298075960_8274\&SEARCH SCREEN=CITATIONS\&SEARCHCONFIG=var_spell. cfg \&DISPLAY=AUTHOR>. Acesso em: 12 dez. 2010.

OWENS, J. A. Concepts of Pitch in English Music Theory, c. 1560-1640. In: JUDD, C. C. (Ed.). Tonal Structures in Early Music. New York and London: Garland, 1998. p. 183-246.

PLAYFORD, John. An introduction to the skill of musick. London: John Playford, 1655. 56p.

SIMPSON, Christopher. A compendium of practical musick in five parts. London: William Godbid, 1667. 176p. 